\title{
Analysis of clinical and genetic characteristics of Chinese children with congenital hyperinsulinemia that is spontaneously relieved
}

\author{
Zi-di Xu ${ }^{1} \cdot$ Pei-pei Hui ${ }^{2} \cdot$ Wei Zhang ${ }^{3} \cdot$ Qiao Zeng $^{1} \cdot$ Lin Zhang $^{1} \cdot$ Min Liu ${ }^{1} \cdot$ Jie Yan ${ }^{1} \cdot$ Yu-jun Wu ${ }^{1} \cdot$ Yan-mei Sang ${ }^{1}$
}

Received: 26 July 2020 / Accepted: 9 December 2020 / Published online: 27 January 2021

(c) The Author(s) 2021

\begin{abstract}
Objective This study aimed to analyze the clinical and genetic characteristics of Chinese children with congenital hyperinsulinemia (CHI) that is spontaneously relieved.

Methods The patient group comprised 200 children with CHI that were treated at the Beijing Children's Hospital from January 2006 to December 2018. The patients were divided into two groups according to their prognosis: the spontaneous remission group $(n=92)$ and the nonspontaneous remission group $(n=108)$. The clinical characteristics, pathogenic genes, diagnosis and treatment process, and follow-up data of both groups were analyzed retrospectively.

Results Of the 200 children with CHI, 92 achieved spontaneous remission. The age of spontaneous remission was between one month and nine years, and 47 of the children were relieved before the age of one year. The median age of onset was 85 days (range: 1-2825 days) in the spontaneous remission group and 2 days (range: 1-210 days) in the nonspontaneous remission group $(P<0.05)$. The mean birth weight was $3.44 \pm 0.76 \mathrm{~kg}$ for the spontaneous remission group and $3.95 \pm$ $0.75 \mathrm{~kg}$ for the nonspontaneous remission group $(P<0.05)$. Of the 92 children in the spontaneous remission group, 65 were treated with diazoxide with effective rate of $81.5 \%$ (53/65). In 12 cases in which diazoxide treatment failed, octreotide was used with an effective rate of $83.3 \%$ (10/12). Of the 108 children in the nonspontaneous remission group, 88 were treated with diazoxide with an effective rate of $43.2 \%$ (38/88), and 29 children were treated with octreotide with an effective rate of $48.28 \%$ (14/29). Of the 30 children in the spontaneous remission group that underwent mutation analysis of CHI-related pathogenic genes, 10 children $(10 / 30,33.3 \%)$ carried mutations. Of the 48 children in the nonspontaneous remission group that underwent mutation analysis of CHI-related pathogenic genes, 37 children $(37 / 48,77.1 \%)$ were found to carry mutations. All of the differences in the indices mentioned above were statistically significant.

Conclusions The rate of spontaneous remission of CHI was significantly higher in children with late age of CHI onset, light birth weight, effective diazoxide treatment, and no common pathogenic gene mutations. Spontaneous remission was also possible for a small number of children that carried mutations in the ABCC and KCNJ11 genes and in whom diazoxide treatment failed.
\end{abstract}

Keywords Congenital hyperinsulinemia $\cdot$ Self-spontaneous remission $\cdot$ Diazoxide $\cdot$ Octreotide $\cdot$ Pathogenic gene

Supplementary information The online version of this article (https:// doi.org/10.1007/s12020-020-02585-x) contains supplementary material, which is available to authorized users.

Yan-mei Sang

sang_yanmei8569@163.com

1 Department of Endocrinology, Genetics and Metabolism, Beijing Children's Hospital, Capital Medical University, National Center for Children's Health, 100045 Beijing, China

2 Department of Pediatrics, Affiliated Hospital of Xuzhou Medical University, Xuzhou 221000, China

3 Medizinische Klinik und Poliklinik IV, Klinikum der Universität München, Ludwig-Maximilians-Universität München, Munich 80336, Germany

\section{Introduction}

Congenital hyperinsulinism (CHI) is a genetically heterogeneous disease in which intractable, persistent hypoglycemia is induced by excessive insulin secretion and an increase in serum insulin concentrations [1]. CHI is characterized by Intractable hypoglycemia and relative hyperinsulinemia that is not commensurate with the blood glucose level, and it is a major cause of persistent and recurrent hypoglycemia in infants and children. To date, at least 14 gene mutations have been found to be associated with CHI, corresponding to 13 genetic types [1-3]. The clinical characteristics, treatment plan, therapeutic effect, 
and prognosis are different for children with different types of CHI. By far the most common genetic cause of $\mathrm{HI}$ is inactivating mutations in ABCC8 and KCNJ11, which encode the two subunits of the pancreatic beta cell ATPsensitive $\mathrm{K}+(\mathrm{KATP})$ channel [3]. Dominant mutations may be inherited from a parent or can arise spontaneously (i.e., de novo). Hyperinsulinism caused by dominant mutations in ABCC8 and KCNJ11may be milder and diazoxide-responsive, but some of the dominant defects may be as severe and diazoxide-unresponsive as hyperinsulinism caused by biallelic recessive KATP mutations [4]. Recent research has shown that, for some children with $\mathrm{CHI}$, the hypoglycemic symptoms are gradually relieved with increasing age. In this study, 200 Chinese children with CHI were included as subjects and divided into a spontaneous remission group and a nonspontaneous remission group. The clinical and genetic characteristics of the children in each group were summarized and analyzed in order to more comprehensively understand and evaluate the prognosis of $\mathrm{CHI}$ and provide a scientific basis for the precise formulation of a treatment plan for $\mathrm{CHI}$.

\section{Methods}

\section{Subjects}

A total of 200 children with $\mathrm{CHI}$ that were treated at the Beijing Children's Hospital from January 2006 to December 2017 were included as the subjects, along with their parents. Out of these patients, 92 children achieved spontaneous remission during the follow-up period. All of the children included in this study were diagnosed with $\mathrm{CHI}$ after they were admitted to the hospital. The specific diagnostic criteria were as follows: (1) hyperinsulinemia (plasma insulin >2 uIU/mL), (2) hypolipidemia (plasma free fatty acid $<1.5 \mathrm{mmol} / \mathrm{L}$ ), (3) hypoketonemia (serum $\beta$-hydroxybutyric acid $<2.0 \mathrm{mmol} / \mathrm{L}$ ), and (4) blood glucose changes $>30 \mathrm{mg} / \mathrm{dL}$ during a glucagon test with $1 \mathrm{mg}$ intravenous blood [5]. The study was approved by the ethics committee of Beijing Children's Hospital, Capital Medical University (2014-6). The parents of all participants were informed of the purpose of this study and gave their written informed consent prior to recruitment.

\section{Diagnosis and treatment process}

After being diagnosed with CHI, 153 of the 200 children were treated with diazoxide, with an initial dose of $5 \mathrm{mg} /$ $(\mathrm{kg} \cdot \mathrm{d})$ administered orally two to three times per day. The dose was gradually increased according to the patient's condition, with a maximum dose of $15 \mathrm{mg} /(\mathrm{kg} \cdot \mathrm{d})$. When the blood glucose levels returned to normal levels, the dosage of diazoxide was gradually decreased and eventually the administration was discontinued. Diuretic hydrochlorothiazide $(1-2 \mathrm{mg} /[\mathrm{kg} \cdot \mathrm{d}]$, administered orally two to three times per day) was used to prevent the side effects of water and sodium retention. Potassium chloride (1-2 $\mathrm{mL}$ of $10 \%$ potassium chloride/[kg.d], administered orally three times per day) was used to prevent hypokalemia caused by diuretics.

Of the 200 children, 41 children were treated with octreotide. The initial dose of octreotide was $5 \mu \mathrm{g} /(\mathrm{kg} \cdot \mathrm{d})$, subcutaneously injected three to four times per day; the dose was adjusted according to the patient's blood glucose levels. The maximum recommended dose of octreotide is generally $20 \mu \mathrm{g} /(\mathrm{kg} \cdot \mathrm{d})$.

\section{Genetic analysis}

\section{Sample acquisition}

After informed consent was obtained, genetic sequencing and analysis was conducted on 78 of the children and their parents. Three $\mathrm{mL}$ of venous blood was collected from the children and their parents and treated with the anticoagulant ethylenediaminetetraacetic acid. The genomic DNA of the patients and their families was extracted using the BloodGen Midi kit (CWBIO, China). All operations were carried out in strict accordance with the kit instructions.

\section{Second-generation sequencing technology}

A capture probe was used for the targeted capture of the exon regions of the genes associated with hypoglycemia, as identified by previous research and the Online Mendelian Inheritance in Man (OMIM) database. The chip used in the total exon capture was from the SeqCap EZ MedExome enrichment kit (Roche, Switzerland). The sequencing operation flow was standardized using the HiSeq $\mathrm{X}$ Ten platform (Illumina, Inc., San Diego, CA, USA).

\section{First-generation sequencing (Sanger method) for validation}

If the second-generation sequencing technology revealed that a child carried the mutations associated with $\mathrm{CHI}$ in the ABCC8, KCNJ11, GLUD1, GCK, HNF4 $\alpha$, HNF1 $\alpha$, HADH, UCP2, SLC16A1, HK1, PGM1, or CACN $\alpha 1 D$ genes, primers were designed according to the sequence of the verified loci in the gene. The DNA was amplified using the polymerase chain reaction (PCR) and sequenced with the ABI 3730 DNA analyzer (Applied Biosystems, Waltham, MA, USA). The primers used for sequencing were the same as those used in PCR amplification. The gene sequences were analyzed and compared using the DNASTAR Lasergene software (Madison, Wisconsin, USA). 
Table 1 General clinical data of $92 \mathrm{CHI}$ children that was spontaneously relieved

\begin{tabular}{lc}
\hline Clinical characteristics & Results $(n=92)$ \\
\hline Male, $n(\%)$ & $49(53.3 \%)$ \\
Birth weight, kg (range) & $24.44(26.1 \%)$ \\
Macrosomia, $n(\%)$ & \\
Age of onset, $n(\%)$ & $41(44.6 \%)$ \\
$\quad<1$ month & $23(25.0 \%)$ \\
$1-6$ months & $28(30.4 \%)$ \\
$>6$ months & \\
Age of spontaneous remission & $2(2.2 \%)$ \\
$\quad<1$ month & $8(8.7 \%)$ \\
$1-3$ months & $37(40.2 \%)$ \\
3 months-1 year & $30(32.6 \%)$ \\
$1-3$ years & $15(16.3 \%)$ \\
$>3$ years & $65(70.7 \%)$ \\
Treatment of diazoxide $n(\%)$ & $53(81.5 \%)$ \\
Effective in diazoxide $n(\%)$ & $30(32.6 \%)$ \\
Gene sequencing $n(\%)$ & $10(33.3 \%)$ \\
Cases carried mutations of CHI pathogenic & \\
genes $n(\%)$ & \\
\hline
\end{tabular}

\section{Database analysis}

Non-pathogenic mutations were excluded based on the Single-Nucleotide Polymorphism Database and the 1000 Genomes Project. The OMIM database following the American College of Medical Genetics and Genomics criteria(http://acmg.cbgc.org.cn/doku.php?id=start). The Human Gene Mutation Database, and the databases from the National Center for Biotechnology Information were used to determine whether a mutation had been previously reported.

\section{Protein structure prediction software}

The effects of gene mutations on protein structure were predicted using the Protein Variation Effect Analyzer software and the Sorting Intolerant from Tolerant algorithm.

\section{Follow-up method}

After discharge, the patients were followed up for a period of one to nine years. During follow-up visits, fasting and 2$\mathrm{h}$ postprandial blood glucose levels were regularly monitored; the therapeutic effect of drugs was evaluated, adverse drug reactions were monitored, and the dosage of drugs was regulated; the inducement of hypoglycemia during the treatment was recorded; and the time and age at which hypoglycemia was spontaneously relieved was recorded.
The spontaneous remission of hypoglycemia symptoms was defined as blood glucose levels maintained at $\geq 60 \mathrm{mg} / \mathrm{dL}$ for at least three months without treatment or after drug discontinuation [6]. The spontaneous remission was confirmed by an overnight fasting test. Using this standard for spontaneous remission, the 200 children were divided into two groups: the spontaneous remission group $(n=92)$ and the nonspontaneous remission group $(n=108)$.

\section{Statistical analysis}

The clinical data of the spontaneous and nonspontaneous remission groups were analyzed and compared. The measurement data were expressed as the mean \pm standard deviation $(\mathrm{x} \pm \mathrm{SD})$. The skewed data were expressed as the median (range). SPSS 19.0 software (IBM Corp., Chicago, USA) was used for data processing. The statistical methods included the chi-square test and the analysis of variance. $P<0.05$ was considered statistically significant for all results.

\section{Results}

\section{Clinical characteristics}

Of the 200 children with CHI, 117 were boys and 83 children were girls. Regarding birth weight, 78 of the children were macrosomic, 113 children had a normal birth weight, and 9 had a low-birth weight. Regarding the age of onset, 132 children developed the disease within one month of birth, 39 children developed the disease between one and six months after birth, and 29 children developed the disease more than six months after birth. Among the $200 \mathrm{CHI}$ children, 142 presented with the initial symptom of seizures; 29 presented with drowsiness; 2 presented with excessive sweating; 1 presented with diarrhea; 1 presented with fatigue. The rest 25 had no symptoms. No other symptoms related to HI were observed. None of them had any risk factors for transient hyperinsulinism, such as small for dates or perinatal stress. None of them were diagnosed with such as Beckwith Weidemann Syndrome, Kabuki, Turner or Sotos.

Of the 92 children that achieved spontaneous remission, 49 were boys and 43 were girls. The children had birth weights of $1.5-6.0 \mathrm{~kg} ; 24$ of the children were macrosomic, 61 had a normal birth weight, and 7 had a low-birth weight. The age of onset ranged from one day to seven years and nine months: The disease developed within one month of birth in 41 children, between one and six months after birth in 23 children, and more than six months after birth in 29 children (Table 1). 
Table 2 Carrying of pathogenic gene mutations in 10 children with CHI that was spontaneously relieved

\begin{tabular}{|c|c|c|c|c|c|}
\hline Case no & Gene mutation & Amino acid mutation & Source of mutation & Curative effect of diazoxide & Age of spontaneous remission \\
\hline \multicolumn{6}{|c|}{$A B C C 8$ gene } \\
\hline 5 & c. $3650 \mathrm{G}>\mathrm{A}$ & p.R1217K & Maternal genetic & Effective & 1 year old \\
\hline 10 & c. $4513 \mathrm{G}>\mathrm{C}$ & p.D1505H & de novo & Invalid & 1 year old \\
\hline 39 & c. $3455 \mathrm{C}>\mathrm{T}$ & p.A1152V & Maternal genetic & Unused & 5 months \\
\hline 42 & $\begin{array}{l}\text { c. } 1919 \mathrm{C}>\mathrm{T} \\
\text { c. } 3586 \mathrm{C}>\mathrm{T}\end{array}$ & $\begin{array}{l}\text { p.A640V } \\
\text { p.Q1196X }\end{array}$ & $\begin{array}{l}\text { de novo s } \\
\text { Paternal genetic }\end{array}$ & Effective & 1 year old \\
\hline 45 & $\begin{array}{l}\text { c. } 3000 \mathrm{C}>\mathrm{A} \\
\text { c. } 824 \mathrm{G}>\mathrm{A}\end{array}$ & $\begin{array}{l}\text { p.C1000X } \\
\text { p.R275Q }\end{array}$ & $\begin{array}{l}\text { Paternal genetic } \\
\text { de novo }\end{array}$ & Effective & 2 years old \\
\hline 49 & c. $4478 \mathrm{G}>\mathrm{A}$ & p.R1493Q & Paternal genetic & Invalid & 10 months \\
\hline 54 & c.12Cdel & Frameshift mutations & Paternal genetic & Invalid & 6 months \\
\hline 64 & c. $1484 \mathrm{G}>\mathrm{A}$ & p.R495Q & Paternal genetic & Unused & 1 year and 9 months \\
\hline \multicolumn{6}{|c|}{ GLUD1 gene } \\
\hline 92 & c. $1388 \mathrm{~A}>\mathrm{G}$ & p.N463S & de novo & Unused & 9 years old \\
\hline \multicolumn{6}{|c|}{ SLC16A1 gene } \\
\hline 91 & c. $1486 \mathrm{C}>\mathrm{T}$ & p. $496 \mathrm{E}>\mathrm{K}$ & Maternal genetic & Effective & 4 years and 2 months \\
\hline
\end{tabular}

\section{Diagnosis and treatment effect}

Treatment with diazoxide and octreotide was considered to be ineffective when the maximum dose was used for five days and the blood glucose levels of children that had fasted for more than $8-10 \mathrm{~h}$ could not be maintained above $70 \mathrm{mg} /$ $\mathrm{dL}[7,8]$.

Of the 200 children diagnosed with CHI, 153 were treated with diazoxide after they were diagnosed with CHI. The blood glucose levels of 91 of these children gradually recovered to normal levels after being treated with diazoxide, suggesting that the treatment with diazoxide was effective for these children. The blood glucose levels of the other 62 children did not significantly improve after being treated with diazoxide at the maximum recommended dose of $15 \mathrm{mg} /(\mathrm{kg} \cdot \mathrm{d})$; this suggests that the treatment with diazoxide was ineffective for these children. Out of the group of 200 children, 41 children were treated with octreotide. The treatment with octreotide was effective for 24 children and ineffective for 17 children.

Of the 92 children with $\mathrm{CHI}$ that was spontaneously relieved, 27 cases relieved spontaneously through intensive feeding which were not treated with any medicine such as diazoxide and the other 65 were treated with diazoxide (53 were responsive to diazoxide). Octreotide was used in 12 children who were unresponsive to diazoxide. All the spontaneous remission group $\mathrm{CHI}$ did not underwent surgery.

Of the 108 children in the nonspontaneous remission group, 88 were treated with diazoxide( 38 were responsive to diazoxide). Among the rest 50 children who were unresponsive to diazoxide, 25 were treated with Octreotide and 15 underwent surgery. Of the 15 children treated with surgery, 11 with focal lesion received partial pancreatectomy and the other four patients received total pancreatectomy. In the nonspontaneous remission group, besides the children who were treated with diazoxide, 20 were treated with $(n=1)$ or without $(n=19)$ Octreotide. None of them underwent surgery.

\section{Results of the genetic analysis}

Of the 108 children diagnosed with $\mathrm{CHI}$ that was nonspontaneously relieved, 48 underwent mutation analysis of CHI-related pathogenic genes, and 37 were found to carry mutations of the known pathogenic genes (Supplemental Table 1):

(1) Thirty-one children carried mutations in the ABCC and KCNJ11 genes, of which 6 carried the compound heterozygous mutation in the $\mathrm{ABCC} 8$ gene, 1 carried both $\mathrm{ABCC} 8$ and $\mathrm{KCNJ} 11$ gene mutations, and 24 carried single ABCC8 or KCNJ11 gene mutations. Of the single gene mutations, 17 were paternal inherited, 2 were maternal inherited, 4 were de novo mutations., and the inheritance of the other one was unknown.

(2) Three children carried mutations in the GLUD1 gene.

(3) Three children carried other rare pathogenic mutations in the HNF4A and HADH genes.

Of the 92 children with $\mathrm{CHI}$ that was spontaneously relieved, 30 underwent mutation analysis of CHI-related pathogenic genes, and 10 were found to carry mutations of the known pathogenic genes (Table 2):

(1) Eight children carried mutations in the ABCC8 gene, of which two carried the compound heterozygous mutation in the $\mathrm{ABCCA} 8$ gene, and six carried single $\mathrm{ABCC} 8$ gene mutations. Of the single gene mutations, three were paternal 
Table 3 General clinical characteristics and prognosis of children with $\mathrm{CHI}$ in the spontaneous remission group and the nonspontaneous remission group

\begin{tabular}{|c|c|c|c|c|}
\hline & $\begin{array}{l}\text { Spontaneous } \\
\text { remission group }\end{array}$ & $\begin{array}{l}\text { Non spontaneous } \\
\text { remission group }\end{array}$ & $\begin{array}{l}\text { Statistical } \\
\text { results }\end{array}$ & $P$ \\
\hline Birth weight (kg) & $3.44 \pm 0.76$ & $3.95 \pm 0.74$ & $\mathrm{~F}=19.35$ & 0.000 \\
\hline Age of onset (days) & $85(1-2825)$ & $2(1-210)$ & $\mathrm{F}=18.44$ & 0.000 \\
\hline $\begin{array}{l}\text { Effective rate of } \\
\text { diazoxide, } \mathrm{n}(\%)\end{array}$ & $53 / 65(81.54)$ & $38 / 88(43.18)$ & $\chi^{2}=22.82$ & 0.000 \\
\hline $\begin{array}{l}\text { Effective rate of } \\
\text { octreotide, } \mathrm{n}(\%) \\
\text { Probability of carrying gene } \\
\text { mutation, n }(\%)\end{array}$ & $\begin{array}{l}10 / 12(83.33) \\
10 / 30(33.33)\end{array}$ & $\begin{array}{l}14 / 29(48.28) \\
37 / 48(77.08)\end{array}$ & $\begin{array}{l}\chi^{2}=4.30 \\
\chi^{2}=14.76\end{array}$ & $\begin{array}{l}0.038 \\
0.000\end{array}$ \\
\hline
\end{tabular}

The data is expressed as median (range) or mean \pm standard deviation inherited, two were maternal inherited, and one was a de novo mutation.

(2) One child carried a mutation in the GLUD1 gene.

(3) One child carried a mutation in SLC16A1 gene.

\section{Follow-up results for the 200 children with $\mathrm{CHI}$}

Of the 200 children with $\mathrm{CHI}, 92$ were able to maintain normal blood glucose levels for more than three months without any further treatment and after the discontinuation of drugs; this suggests that the hypoglycemic symptoms of the patients in this group were spontaneously alleviated. The age of spontaneous remission for the 92 children was between one month and nine years, and 47 of the children were relieved before the age of one year. Table 3 compares the children in the spontaneous and nonspontaneous remission groups in terms of their general clinical characteristics and prognoses. The mutations identified in the nonspontaneous remission group are shown in Supplementary Table 1.

\section{Discussion}

As clinical research on $\mathrm{CHI}$ intensifies, more and more attention is being paid to the phenomenon of spontaneous remission in patients with CHI. A patient is characterized as having achieved spontaneous remission of $\mathrm{CHI}$ when normal blood glucose levels can be maintained for at least three months without medication, or medication has been stopped medication and further medical or surgical treatment is not needed. In 2011, the Banerjee team in the United Kingdom studied 101 pediatric patients with $\mathrm{CHI}$ and found that spontaneous remission was achieved by approximately $47.5 \%$ of the patients [5]. In a 2013 study, the Italian Sogno Valin team studied 33 children with $\mathrm{CHI}$ and found a spontaneous remission rate of $27.3 \%$ [9]. A study in 2016 by the Spanish Martinez team found a spontaneous remission rate of $24 \%$ for 50 children with $\mathrm{CHI}$ [10]. In the present study, the long-term follow-up of 200 Chinese children with $\mathrm{CHI}$ found a spontaneous remission rate of
$46 \%(92 / 200)$, a result that is basically consistent with the results of the Banerjee team [6]. This suggests that spontaneous remission is an important outcome of patients with $\mathrm{CHI}$, and it is necessary to study the spontaneous remission of patients with different ethnic backgrounds.

For the 92 children considered in this study that achieved spontaneous remission, the ages of onset of $\mathrm{CHI}$ ranged from one day to seven years and nine months, and the birth weights ranged between 1.5 and $6.0 \mathrm{~kg}$. The distributions of the ages of onset and birth weights had no special characteristics. When the children in the spontaneous and nonspontaneous remission groups were compared in terms of birth weight and age of onset, it was found that the probability of spontaneous remission was significantly higher for children with a lighter birth weight and a later age of onset $(P<0.05$, Table 3$)$. Some children with $\mathrm{CHI}$ with a low gestational age may have temporary diseases [11]. In 2017, the Sakakibara A team in Japan reported that low-birthweight infants were more likely to achieve relief of hypoglycemia [12]. However, the Banerjee team in the UK found that there was no significant difference in birth weight between the children in the spontaneous and nonspontaneous remission groups [6], which suggests that ethnic differences may be relevant to spontaneous remission.

Previous work has reported large differences in the age of spontaneous remission of children with CHI: While remission was on average achieved at 1-5 years of age [13], the latest age of remission was 12 years [14]. Working in the UK, the Banerjee team found that the earliest age of remission was six days after birth, while the latest age of remission was seven and a half years [6]. In addition, $25 \%$ of the children they studied achieved spontaneous remission within one month of birth, $48 \%$ achieved spontaneous remission within three months, and $75 \%$ achieved spontaneous remission within one year. The present study found that the age of spontaneous remission was between one month and nine years, where $51.1 \%$ (47/92) of the children achieved spontaneous remission within one year, and $75 \%$ achieved spontaneous remission within two years. Thus, the mean age of remission found in this study was slightly higher than the mean age reported in the previous literature. 
Of the 200 children with CHI that took part in this study, 153 were treated with diazoxide after they were diagnosed with CHI. Out of the children treated with diazoxide, 91 achieved an effective outcome while 62 did not. Sixty-five of the 92 children in the spontaneous remission group were treated with diazoxide, and 53 achieved an effective outcome while 12 did not. The effective rate of diazoxide was $81.54 \%(53 / 65)$ in the spontaneous remission group, which was significantly higher than the effective rate of the nonspontaneous remission group $(38 / 88,43.18 \% ; P<0.05)$. This shows that the rate of spontaneous remission was significantly higher in patients that underwent effective diazoxide treatment, a finding that is consistent with the previous literature [6].

Forty-one of the 200 children enrolled in this study were treated with octreotide, of which 24 achieved an effective outcome while 17 did not. Octreotide was used to treat 12 of the 92 children in the spontaneous remission group, of which 10 achieved an effective outcome (10/12, 83.33\%). This effective rate was significantly higher than the effective rate of the nonspontaneous remission group (14/29, $48.28 \% ; P<0.05)$. This shows that patients with $\mathrm{CHI}$ that achieved an effective outcome from octreotide treatment had a higher rate of spontaneous remission. There is no previous literature addressing the relationship between spontaneous remission and the outcome of octreotide treatment.

Of the 200 children with $\mathrm{CHI}, 78$ underwent mutation analysis of CHI-related pathogenic genes, and it was found that 47 children carried mutations of these genes. Of the 31 children that did not have gene mutation, 20 achieved spontaneous remission, at a rate of $64.5 \%$ (20/31). This rate is higher than the rates reported by Martinez team in Spain (41\%) and the Sogno Valin team in Italy (44\%), and it is basically consistent with the rate reported by the Banerjee team in the UK $(65 \%)[9,10]$.

When the children in the spontaneous and nonspontaneous remission groups were compared, it was found that the carrier rate of gene mutation was significantly lower in the spontaneous remission group $(10 / 30,33.33 \%)$ than in the nonspontaneous remission group (37/48, 77.08\%; $P<$ $0.05)$. This suggests that the rate of spontaneous remission is higher in children without the mutation of CHI-related pathogenic gene, a finding that is consistent with the conclusions of the Banerjee team in the UK [9, 10].

Previous studies considered the rate of gene mutations in children with $\mathrm{CHI}$ that achieved spontaneous remission and in children that did not achieve spontaneous remission but were effectively treated with drugs. Approximately 15-17\% of these children were found to carry mutations in one or both of the ABCC and KCNJ11 genes, while another $4-6 \%$ carried other types of gene mutations, including mutations in the GLUD1, GCK, and HNF4 $\alpha$ genes [6, 15-17]. In the present study, genetic analysis was carried out on 30 of the 92 patients in the spontaneous remission group. According to this analysis, eight children carried ABCC8 gene mutations $(8 / 30,26.67 \%)$, one child carried a mutation of the GLUD1 gene $(1 / 30,6.7 \%)$, and one other child carried a mutation of the SLC16A1 gene $(1 / 30,6.7 \%)$. These proportions are higher than those reported in the previous literature; this may be due to a difference in the sample size.

The previous literature found that children with $\mathrm{CHI}$ that carried the maternal heterozygous mutation of the ABCC8 gene were most likely to achieve spontaneous remission, with rates of remission of $67-100 \%$ [6, 14]. In the present study, 4 of the 78 children that underwent genetic analysis were found to carry the maternal heterozygous mutation of the ABCC 8 gene, and 2 of those children achieved spontaneous remission; the resulting rate of $50.0 \%(2 / 4)$ is consistent with the rates reported in the previous literature [6, 14]. However, a higher remission rate for individuals with maternally inherited mutation in ABCC8 compared to those with other types of mutations is probably due to the fact that $\mathrm{ABCC} 8$ is dominant mutation and the others may be mild mutations. Of the 20 children that were found to carry the paternal heterozygous mutation of the ABCC8 gene, 3 achieved spontaneous remission; the resulting rate of $15.0 \%(3 / 20)$ is slightly lower than the rate reported in the previous literature $[13,17]$. Of the 8 children that were found to carry the compound heterozygous mutation in the ABCC8 gene, 2 were spontaneously alleviated, giving a remission rate of $25.0 \%(2 / 8)$. These results and those of previous work suggest that the detailed genetic analysis of children can be used as reference for prediction of spontaneous remission. However, adequate caution needs to be exercised when taking children off medication to ensure blood glucose levels are maintained within the safe range to avoid irreversible brain damage. Different pathogenic genes and genetic patterns are also shown to be associated with different rates of spontaneous remission. It is worth noting that the results of this study and previous work suggest that spontaneous remission may still be possible for children with $\mathrm{ABCC}$ and $\mathrm{KCNJ} 11$ gene mutations that do not achieve an effective outcome from diazoxide treatment. For these children, medical treatment must be insisted upon, and, after a longer follow-up period, it should be decided whether a pancreatectomy should be performed.

The exact mechanism of spontaneous remission in children with CHI is still not clear. Early work suggested that it is related to excessive insulin secretion, which leads to the excessive apoptosis of pancreatic $\beta$-cells. However, subsequent studies argued that spontaneous remission may be a process of functional change. 18F-L-DOPA PET/CT pancreatic scanning technology has revealed that the uptake of L-DOPA by pancreatic $\beta$-cells can still be observed in the early stage of spontaneous remission of focal pancreatic 
lesions. This suggests that there is no abnormality in the insulin secretion ability and that pancreatic $\beta$-cells in the lesion area lose the ability to recognize calcium ions. This mechanism may be similar to the long-term use of sulfonylureas to treat diabetic patients, which ultimately leads to a decrease in the insulin secretion function of pancreatic $\beta$-cells. Thus, the current view is that while spontaneous remission may be related to the functional shut down of insulin secretion by pancreatic $\beta$-cells, it is not necessarily accompanied by the abnormal apoptosis of pancreatic $\beta$-cells $[11,18]$.

In summary, the results of the present study suggest that with increasing age and need for insulin, the spontaneous remission of CHI can be achieved by some children. The rate of spontaneous remission was found to be significantly higher in children with a late age of CHI onset, a light birth weight, no mutations in CHI-related pathogenic genes, and a successful response to diazoxide treatment. In addition, there is the possibility of spontaneous remission in a small number of children that carry $\mathrm{ABCC}$ and $\mathrm{KCNJ} 11$ gene mutations and in whom diazoxide treatment has failed. Because there was found to be a large range in the age of spontaneous remission, the length of follow-up should be extended for children with $\mathrm{CHI}$, and surgical treatment should be carefully selected for those children that fail to respond to medical treatment, in order to minimize the chances of surgical complications.

Acknowledgements We would like to acknowledge the hard and dedicated work of all the staff that implemented the intervention and evaluation components of the study.

Funding This work was supported by Beijing Municipal Science \& Technology Commission, No. Z141107002514142. Baoding Selffinancing Project of Science and Technology Plan, No. 1951ZF076

\section{Compliance with ethical standards}

Conflict of interest The authors declare that they have no conflict of interest.

Ethical approval All applicable international, national, and/or institutional guidelines for the care and use of animals were followed. The study was approved by the ethics committee of Beijing Children's Hospital, Capital Medical University(2014-6).

Informed consent Informed consent was obtained from all individual participants included in the study.

Publisher's note Springer Nature remains neutral with regard to jurisdictional claims in published maps and institutional affiliations.

Open Access This article is licensed under a Creative Commons Attribution 4.0 International License, which permits use, sharing, adaptation, distribution and reproduction in any medium or format, as long as you give appropriate credit to the original author(s) and the source, provide a link to the Creative Commons license, and indicate if changes were made. The images or other third party material in this article are included in the article's Creative Commons license, unless indicated otherwise in a credit line to the material. If material is not included in the article's Creative Commons license and your intended use is not permitted by statutory regulation or exceeds the permitted use, you will need to obtain permission directly from the copyright holder. To view a copy of this license, visit http://creativecommons. org/licenses/by/4.0/.

\section{References}

1. O.R. Cabezas, S.E. Flanagan, H. Stanescu, E. García-Martínez, R. Caswell, H. Lango-Allen et al. Polycystic kidney disease with hyperinsulinemic hypoglycemia caused by a promoter mutation in phosphomannomutase 2. J. Am. Soc. Nephrol. 28(8), 2529-39 (2017)

2. S. Galcheva, S. Al-Khawaga, K. Hussain, Diagnosis and management of hyperinsulinaemic hypoglycaemia. Best. Pr. Res Clin. Endocrinol. Metab. 32(4), 551-73 (2018)

3. Z.D. Xu, W. Zhang, M. Liu, H.M. Wang, P.P. Hui, X.J. Liang et al. Analysis on the pathogenic genes of 60 Chinese children with congenital hyperinsulinemia. Endocr. Connect 7(12), 1251-61 (2018)

4. D.D. De León, C.A. Stanley, Determination of insulin for the diagnosis of hyperinsulinemic hypoglycemia. Best. Pract. Res. Clin. Endocrinol. Metab. 27(6), 763-9 (2013). https://doi.org/10. 1016/j.beem.2013.06.005

5. I. Banerjee, M. Skae, S.E. Flanagan, L. Rigby, L. Patel, M. Didi et al. The contribution of rapid $\mathrm{K}_{\mathrm{ATP}}$ channel gene mutation analysis to the clinical management of children with congenital hyperinsulinism. Eur. J. Endocrinol. 164(5), 733-40 (2011)

6. J.Y. Tung, K. Boodhansingh, C.A. Stanley, D.D. De León, Clinical heterogeneity of hyperinsulinism due to HNF1A and HNF4A mutations. Pediatr. Diabetes 3, 1-7 (2018)

7. K. Lord, De León, D. Diva, Monogenic hyperinsulinemic hypoglycemia: current insights into the pathogenesis and management. Int. J. Pediatr. Endocrinol. 2013(1), 3 (2013)

8. R. Martínez, C. Fernández-Ramos, A. Vela, T. Velayos, A. Aguayo, I. Urrutia,Spanish Congenital Hyperinsulinism Group et al., Clinical and genetic characterization of congenital hyperinsulinism in Spain. Eur. J. Endocrinol. 174(6), 717-26 (2016).

9. P. Sogno Valin, M.C. Proverbio, C. Diceglie, A. Gessi, S. di Candia, B. Mariani et al. Genetic analysis of italian patients with congenital hyperinsulinism of infancy. Horm. Res Paediatr. 79(4), 236-42 (2013)

10. I. Banerjee, M. Salomon-Estebanez, P. Shah, J. Nicholson, K.E. Cosgrove, M.J. Dunne, Invited review therapies and outcomes of congenital hyperinsulinisminduced hypoglycaemia. Diabet. Med 36(1), 9-21 (2019)

11. A. Sakakibara, Y. Hashimoto, R. Kawakita, Y. Hosokawa, K. Nagahara, Y. Hasegawa et al. Diagnosis of congenital hyperinsulinism: Biochemical profiles during hypoglycemia. Pediatr. Diabetes 19(2), 259-64 (2018)

12. T. Yorifuji, Y. Hosokawa, R. Fujimaru, R. Kawakita, H. Doi, T. Matsumoto et al. Lasting 18 F-DOPA PET uptake after clinical remission of the focal form of congenital hyperinsulinism. Horm. Res Paediatr. 76(4), 286-90 (2011)

13. K. Mazor-Aronovitch, D. Gillis, D. Lobel, H.J. Hirsch, O. PinhasHamiel, D. Modan-Moses et al. Long-term neurodevelopmental outcome in conservatively treated congenital hyperinsulinism. Eur. J. Endocrinol. 157(4), 491-7 (2007)

14. S.E. Flanagan, R.R. Kapoor, G. Mali, D. Cody, N. Murphy, B. Schwahn et al. Diazoxide-responsive hyperinsulinemic hypoglycemia caused by HNF4A gene mutations. Eur. J. Endocrinol. 162, 987-92 (2010) 
15. C. Bellanné-Chantelot, C. Saint-Martin, M.J. Ribeiro, C. Vaury, V. Verkarre, J.B. Arnoux et al. ABCC8 and KCNJ11 molecular spectrum of 109 patients with diazoxide-unresponsive congenital hyperinsulinism. J. Med. Genet. 47, 752-9 (2010)

16. A. Fernández-Marmiesse, A. Salas, A. Vega, J.R. FernándezLorenzo, J. Barreiro, A. Carracedo, Mutation spectra of ABCC8 gene in Spanish patients with hyperinsulinism of infancy (HI). Hum. Mutat. 27, 214 (2006)
17. T. Yorifuji, R. Kawakita, Y. Hosokawa, R. Fujimaru, K. Matsubara, K. Aizu et al. Efficacy and safety of long-term, continuous subcutaneous octreotide infusion for patients with different subtypes of KATP-channel hyperinsulinism. Clin. Endocrinol. 78(6), 891-7 (2013)

18. T. Yorifuji, Congenital hyperinsulinism_ current status and future perspectives. Ann. Pediatr. Endocrinol. Metab. 19(2), 57-68 (2014) 